\title{
ON SOME IDEALS OF NEST ALGEBRAS
}

\author{
CHENGJUN HOU AND DEGUANG HAN
}

(Communicated by Palle E. T. Jorgensen)

\begin{abstract}
The purpose of this paper is to characterize the relations of some ideals of a nest algebra such as the Jacobson radical $R_{\mathscr{N}}$, Larson strong radical $R_{\mathscr{N}}^{\infty}$, and ideal $\mathscr{G}$, and to describe all the ideals between $R_{\mathscr{N}}$ and $\mathscr{G}$.
\end{abstract}

\section{Preliminaries}

Let $\mathscr{N}$ be a nest (i.e., a totally ordered complete set of projections containing $O$ and $I$ which is closed in the strong operator topology) on a separable Hilbert space $H$. We denote by $\operatorname{Alg} \mathscr{N}$ the algebra of all operators in $B(H)$ that leave invariant every element of $\mathscr{N}$. A nest $\mathscr{N}$ is continuous of its core, which is the von Neumann algebra generated by the elements of $\mathscr{N}$, is a nonatomic von Neumann algebra. $K(H)$ denotes the set of all the compact operators in $B(H)$. We shall call a set $\left\{P_{\alpha} \mid \alpha \in \Lambda\right\}$ of intervals $P_{\alpha}=M_{\alpha}-N_{\alpha}, M_{\alpha}, N_{\alpha} \in$ $\mathscr{N}, N_{\alpha}<M_{\alpha}$, of the nest $\mathscr{N}$, a partition of $\mathscr{N}$ if the intervals are pairwise orthogonal and the sum $\sum_{\alpha \in \Lambda} P_{\alpha}=I$ in the strong topology. Since $H$ is a separable Hilbert space, then every partition is denumerable. Given a nest $\mathscr{N}$, Larson strong radical $R_{\mathscr{N}}^{\infty}$ is the collection of all operators $X$ in $\operatorname{Alg} \mathscr{N}$ for which, given $\varepsilon>0$, there exists a partition $\left\{P_{\alpha} \mid \alpha \in \Lambda\right\}$ of $\mathscr{N}$ such that $\left\|P_{\alpha} X P_{\alpha}\right\|<\varepsilon$ for all $\alpha \in \Lambda$. If we restrict all partitions to be finite sets of intervals, we will obtain the Jacobson radical $R_{\mathscr{N}}$ of $\operatorname{Alg} \mathscr{N}$ [4]. It is clear that $R_{\mathscr{N}}^{\infty}$ always contain $R_{\mathscr{N}}$. The ideals $R_{\mathscr{N}}, R_{\mathscr{N}}^{\infty}$ have been studied by some authors ([3], [4]). In this paper, we will study the ideal $\mathscr{G}$ of $\operatorname{Alg} \mathscr{N}$ and its relations with $R_{\mathscr{N}}$ and $R_{\mathscr{N}}^{\infty}$. Terms and notation not defined here are taken from [4].

Definition 1. If $E_{1}, E_{2}$ are nonzero orthogonal intervals from $\mathscr{N}$ such that $E_{1} B(H) E_{2} \subseteq \operatorname{Alg} \mathscr{N}$, we say $E_{1}, E_{2}$ are strictly ordered and write $E_{1} \ll E_{2}$.

A mutually orthogonal family of intervals is said to be strictly ordered if it is linearly ordered by the relation $\ll$. The length of such a family is its cardinality.

Received by the editors February 7, 1994 and, in revised form, May 4, 1994.

1991 Mathematics Subject Classification. Primary 47D25, 47D15, 47 D47.

Key words and phrases. Nest algebra, ideals, Jacobson radical, Larson strong radical.

This research was supported by NSF of China and YSF of Shandong Province. 
Definition 2. If $A \in \operatorname{Alg} \mathscr{N}$ and $\varepsilon>0$, we define the $\varepsilon$-order of $A$ to be the number

$R_{\varepsilon}(A)=\operatorname{Sup}\{n \mid$ there exists a strictly ordered family $\mathscr{F}$ of length $n$ with $\|E A E\| \geq \varepsilon$ for all $E \in \mathscr{F}\}$.

Definition 3. Let

$$
\begin{aligned}
\mathscr{G} & =\left\{A \in \operatorname{Alg} \mathscr{N} \mid R_{\varepsilon}(A)<+\infty, \text { for every } \varepsilon>0\right\}, \\
\mathscr{G}_{u} & =\left\{A \in \operatorname{Alg} \mathscr{N} \mid \sup _{\varepsilon>0} R_{\varepsilon}(A)<+\infty\right\}, \\
R_{\mathscr{N}}^{\alpha} & =\{A \in \operatorname{Alg} \mathscr{N} \mid E A E=0 \text { for every atom } E \text { of } \mathscr{N}\} .
\end{aligned}
$$

It is easy to verify that $\mathscr{G}, \mathscr{G}_{u}, R_{\mathscr{N}}^{\alpha}$ are ideals of $\operatorname{Alg} \mathscr{N}$.

\section{MAIN RESUlTS}

In the paper [1], it is shown that $\mathscr{G}$ is a norm-closed ideal of $\operatorname{Alg} \mathscr{N}$ which contains $R_{\mathcal{N}}$. In this section, we will study the relations of $\mathscr{G}_{,} \mathscr{G}_{u}, R_{\mathcal{N}}, R_{\mathscr{N}}^{\infty}$ and $R_{\mathscr{N}}^{\alpha}$.

Let $x \otimes y$ denote the rank-one operator acting on $H$ such that $x \otimes y(z)=$ $\langle z, x\rangle y$, for all $z \in H$.

Theorem 1. $\mathscr{G}_{u}$ is closed in the norm topology if and only if $\mathscr{N}$ is a finite nest. Proof. If $\mathscr{N}$ is finite, then $\mathscr{G}_{u}=\mathscr{G}=\operatorname{Alg} \mathscr{N}$. Thus $\mathscr{G}_{u}$ is norm-closed.

Now suppose $\mathscr{G}_{u}$ is a norm-closed ideal; we claim that $\mathscr{N}$ is finite. Otherwise, there exists $P \in \mathscr{N}$ such that $P=P_{-}(P \neq 0)$ or $P=P_{+}(P \neq I)$. We only need to consider the case: $P=P_{-}(P \neq 0)$. Choose $\left\{P_{n}\right\}, P_{n} \in N$, such that $P_{n}<P_{n+1}, P=\lim _{n \rightarrow \infty} P_{n}$ in the strong operator topology. Let $E_{n}=P_{n+1}-P_{n}$, and $x_{n} \in E_{n}$ such that $\left\|x_{n}\right\|=1, n=1,2, \ldots$.

Let $T_{n}=\sum_{k=1}^{n} \frac{1}{k^{2}} x_{k+1} \otimes x_{k}$, and $T=\sum_{k=1}^{\infty} \frac{1}{k^{2}} x_{k+1} \otimes x_{k}$; then $\lim _{n \rightarrow \infty} T_{n}=$ $T$ in the norm topology and $T_{n} \in \mathscr{G}_{u}(n=1,2, \ldots)$. Now we prove that $T \notin \mathscr{G}_{u}$. For arbitrary positive integer $M$, there exists $\varepsilon>0$ such that $\varepsilon<$ $\frac{1}{(2 M-1)^{2}}$. Let $F_{k}=P_{2 k+1}-P_{2 k-1}(k=1,2, \ldots, M)$, thereby, $\left\|F_{k} T F_{k}\right\|=$ $\frac{1}{(2 k-1)^{2}} \geq \frac{1}{(2 M-1)^{2}}>\varepsilon(k=1,2, \ldots, M)$, and $R_{\varepsilon}(T) \geq M$. Since $M$ is arbitrary, we have $\sup _{\varepsilon>0} R_{\varepsilon}(T)=+\infty$. Thus $T \notin \mathscr{G}_{u}$, which contradicts the closedness of $\mathscr{G}_{u}$.

Theorem 2. Let $\mathscr{N}$ be a nest. Then

(1) $R_{\mathscr{N}}^{\alpha} \cap \mathscr{G}=R_{\mathscr{N}}^{\infty} \cap \mathscr{G}=R_{\mathscr{N}}$.

(2) $\mathscr{G}=R_{\mathscr{N}}$ if and only if $\mathscr{N}$ is continuous.

(3) $\mathscr{G} \varsubsetneqq R_{\mathscr{N}}^{\infty}$ if and only if $\mathscr{N}$ is continuous.

(4) $\mathscr{G} \supseteq R_{\mathcal{N}}^{\infty}$ if and only if $\mathscr{N}$ is finite.

For arbitrary nest $\mathscr{N}, \mathscr{G} \neq R_{\mathscr{N}}^{\infty}$.

Proof. (1) The chain $R_{\mathscr{N}} \subseteq R_{\mathscr{N}}^{\infty} \cap \mathscr{G} \subseteq R_{\mathscr{N}}^{\alpha} \cap \mathscr{G}$ is evident. It remains to show that each $X \in R_{\mathscr{N}}^{\alpha} \cap \mathscr{G}$ belongs to $R_{\mathscr{N}}$. Apply Ringrose's Criterion, using the seminorms $i_{N}^{+}(X)$ and $i_{N}^{-}(X)$ (see [4]). Let $\varepsilon>0$ and $N \in \mathscr{N}$. By symmetry it suffices to show that $i_{N}^{+}(X) \leq \varepsilon$. If $N^{+}>N$, then $N^{+}-N$ is an atom and

$$
i_{N}^{+}(X)=\left\|\left(N^{+}-N\right) X\left(N^{+}-N\right)\right\|=0 .
$$

Otherwise there is a sequence $N_{n}$ decreasing to $N$ in $\mathscr{N}$. Suppose that $i_{N}^{+}(X)>\varepsilon$. For each fixed $k,\left(N_{k}-N_{n}\right) X\left(N_{k}-N_{n}\right)$ converges strongly to 
$\left(N_{k}-N\right) X\left(N_{k}-N\right)$, which is greater than $\varepsilon$ in norm. So, by the strong lower semicontinuity of the norm there is a $k^{\prime}$ such that

$$
\left\|\left(N_{k}-N_{k^{\prime}}\right) X\left(N_{k}-N_{k^{\prime}}\right)\right\|>\varepsilon .
$$

Passing to a subsequence, we can assume

$$
\left\|\left(N_{k}-N_{k+1}\right) X\left(N_{k}-N_{k+1}\right)\right\|>\varepsilon
$$

for all $k$. But this means that $R_{\varepsilon}(X)=+\infty$, which is a contradiction.

(2) It is easy to prove that $E \in \mathscr{G}, E \notin R_{\mathscr{N}}^{\alpha}$, for every atom $E$. By (1), $\mathscr{G}=R_{\mathcal{N}}$ if and only if $\mathscr{G} \subseteq R_{\mathscr{N}}^{\alpha}$ if and only if $\mathscr{N}$ is continuous.

For (3), (4), by (1), we can prove them easily.

\section{Proposition 1. Let $\mathcal{N}$ be a nest. Then}

(1) $R_{\mathscr{N}} \subseteq \overline{\mathscr{G}}_{u}$, where the closure is in the norm topology.

(2) $R_{\mathscr{N}} \subset \mathscr{G}_{u}$ if and only if $\mathscr{N}$ is finite.

(3) $\mathscr{G}_{u} \subset R_{\mathscr{N}}$ if and only if $\mathscr{N}$ is continuous.

For arbitrary nest $\mathscr{N}, R_{\mathscr{N}} \neq \mathscr{G}_{u}$.

Proof. (1) Since $R_{\mathscr{N}}=\overline{\operatorname{span}}\left\{P A P^{\perp} \mid P \in \mathscr{N}, A \in \operatorname{Alg} \mathscr{N}\right\}$, where the closure is in the norm topology, and $P A P^{\perp} \in \mathscr{G}_{u}$, for every $P \in \mathscr{N}, A \in \operatorname{Alg} \mathscr{N},(1)$ holds.

(2) If $\mathscr{N}$ is finite, then $R_{\mathscr{N}} \subset \mathscr{G}_{u}=\operatorname{Alg} \mathscr{N}$. If $\mathscr{N}$ is infinite, we have constructed $T \in R_{\mathscr{N}}$, but $T \notin \mathscr{G}_{u}$ in the proof of Theorem 1. This contradicts $R_{\mathscr{N}} \subset \mathscr{G}_{u}$.

(3) If $\mathscr{N}$ is continuous, from Theorem 2 (2), $\mathscr{G}=R_{\mathscr{N}} \supset \mathscr{G}_{u}$. If $\mathscr{N}$ is not continuous, then there exists an atom $E$. Let $x, y \in E$ such that $\|x\|=\|y\|=$ 1 ; then $x \otimes y \notin R_{\mathscr{N}}, x \otimes y \in \mathscr{G}_{u}$. Thus $\mathscr{G}_{u} \nsubseteq R_{\mathcal{N}}$.

In the paper [2], Lance gave a standard form for all ideals containing the radical of a nest algebra, but not in a very explicit manner. In the last theorem of this paper, we will, by the index function of atoms, characterize all the normclosed ideals between $R_{\mathscr{N}}$ and $\mathscr{G}$ in an explicit way.

Let $\left\{E_{\alpha} \mid \alpha \in \Lambda\right\}$ be all the atoms of $\mathscr{N}$. Let

$$
\operatorname{ind}(\alpha)= \begin{cases}0 & \text { if } \operatorname{dim} E_{\alpha}<\infty \\ 1 & \text { if } \operatorname{dim} E_{\alpha}=\infty\end{cases}
$$

denote the index function of $\left\{E_{\alpha} \mid \alpha \in \Lambda\right\}$.

Let $\mathscr{F}=\{\varphi \mid \varphi: \Lambda \rightarrow\{0,1,2\}$ such that $\varphi(\alpha) \leq \operatorname{ind}(\alpha)+1\}$. For $\varphi \in$ $\mathscr{F}, \alpha \in \Lambda$, let

$$
M_{\varphi(\alpha)}= \begin{cases}0 & \text { if } \varphi(\alpha)=0, \\ E_{\alpha} K(H) E_{\alpha} & \text { if } \varphi(\alpha)=1, \\ E_{\alpha} B(H) E_{\alpha} & \text { if } \varphi(\alpha)=2 .\end{cases}
$$

Definition 4. For $\varphi \in \mathscr{F}$, let

$$
\mathscr{D}_{\varphi}=\left\{\begin{array}{l}
T \\
\begin{array}{l}
T=\sum_{\alpha \in \Lambda} T_{\alpha}, T_{\alpha} \in M_{\varphi(\alpha)} \\
\lim \left\|T_{\alpha}\right\|=0
\end{array}
\end{array}\right\},
$$

where $\lim \left\|T_{\alpha}\right\|=0$ means: for every $\varepsilon>0$, set $\left\{\alpha \in \Lambda \mid\left\|T_{\alpha}\right\| \geq \varepsilon\right\}$ is finite, and let

$$
\mathscr{G}_{\varphi}=R_{\mathscr{N}} \oplus \mathscr{D}_{\varphi}
$$


In the next theorem, we will identify $\mathscr{G}$ with $R_{\mathscr{T}} \oplus D_{0}$, where $D_{0}$ is the set of operators which are norm convergent sums of the form $\sum E_{i} T E_{i}$ (with $E_{i}$ an enumeration of the atoms). Clearly, $D_{0}=D_{\varphi_{0}}$, where

$$
\varphi_{0}(\alpha)= \begin{cases}1 & \text { if } \operatorname{ind}(\alpha)=0 \\ 2 & \text { if } \operatorname{ind}(\alpha)=1\end{cases}
$$

Theorem 3. Let $\mathscr{N}$ be a nest. Then

(1) If I is a closed two-sided ideal of $\operatorname{Alg}_{\mathscr{N}}$ in the norm topology and $R_{\mathscr{N}} \subseteq$ $I \subseteq \mathscr{G}$, then there exists $\varphi \in \mathscr{F}$ such that $I=\mathscr{G}_{\varphi}$. In particular, $\mathscr{G}=R_{\mathscr{N}} \oplus \mathscr{D}_{0}$.

(2) For every $\varphi \in \mathscr{F}, \mathscr{G}_{\varphi}$ is a norm-closed two-sided ideal of $\operatorname{Alg} \mathscr{N}$, and $R_{\mathscr{N}} \subseteq \mathscr{G}_{\varphi} \subseteq \mathscr{G}$.

(3) $\mathscr{G}_{\varphi}=\mathscr{G}_{\varphi^{\prime}}$ if and only if $\varphi=\varphi^{\prime}$.

Proof. (1) For every $\alpha \in \Lambda, E_{\alpha} I E_{\alpha}$ is an ideal of $E_{\alpha}(\operatorname{Alg} \mathscr{N}) E_{\alpha}$, which is closed in the norm topology. If ind $(\alpha)=0$, then $E_{\alpha} I E_{\alpha}=0$ or $E_{\alpha} I E_{\alpha}=$ $E_{\alpha} B(H) E_{\alpha}=E_{\alpha} K(H) E_{\alpha}$. If $\operatorname{ind}(\alpha)=1$, then $E_{\alpha} I E_{\alpha}=0$ or $E_{\alpha} I E_{\alpha}=$ $E_{\alpha} K(H) E_{\alpha}$ or $E_{\alpha} I E_{\alpha}=E_{\alpha} B(H) E_{\alpha} \neq E_{\alpha} K(H) E_{\alpha}$. Let

$$
\varphi(\alpha)= \begin{cases}0 & \text { if } E_{\alpha} I E_{\alpha}=0 \\ 1 & \text { if } E_{\alpha} I E_{\alpha}=E_{\alpha} K(H) E_{\alpha}, \\ 2 & \text { if } E_{\alpha} I E_{\alpha}=E_{\alpha} B(H) E_{\alpha} \neq E_{\alpha} K(H) E_{\alpha} .\end{cases}
$$

Then $\varphi \in \mathscr{F}$ and $M_{\varphi(\alpha)} \subseteq I$. By the definition of $\mathscr{G}_{\varphi}$, we have $\mathscr{G}_{\varphi} \subseteq I$. On the other hand, for $T \in I$, let $S=T-\sum_{\alpha \in \Lambda} T_{\alpha}$, where $T_{\alpha}=E_{\alpha} T E_{\alpha}, \alpha \in \Lambda$. Since $T \in \mathscr{G}$, we have $\sum_{\alpha \in \Lambda} T_{\alpha} \in \mathscr{G}$ and $\lim \left\|T_{\alpha}\right\|=0$. Therefore $S \in \mathscr{G}$. Furthermore $E_{\alpha} S E_{\alpha}=0$, for $\alpha \in \Lambda$; then $S \in R_{\mathscr{N}}^{\alpha}$ and so $S \in R_{\mathscr{N}}^{\alpha} \cap \mathscr{G}=R_{\mathcal{V}}$. Thus $T=S+\sum_{\alpha \in \Lambda} T_{\alpha} \in \mathscr{G}_{\varphi}$. Since $T$ is arbitrary, we have $I \subseteq \mathscr{G}_{\varphi}$, which implies that $I=\mathscr{G}_{\varphi}$.

(2) Letting $\varphi \in \mathscr{F}$, by the definition of $\mathscr{G}_{\varphi}$, we know that $R_{\mathscr{V}} \subseteq \mathscr{G}_{\varphi} \subseteq \mathscr{G}$. Let $I$ be the norm-closed two-sided ideal of Alg $\mathscr{N}$ generated by $\mathscr{G}_{\varphi}$. Then $R_{\mathscr{N}} \subseteq I \subseteq \mathscr{G}$. By the proof of (1), we have $I=\mathscr{G}_{\varphi}$. Thus $\mathscr{G}_{\varphi}$ is a two-sided ideal of $\operatorname{Alg} \mathscr{N}$, which is closed in the norm topology.

(3) If $\varphi \neq \varphi^{\prime}$, then there exists $\alpha \in \Lambda$ such that $\varphi(\alpha) \neq \varphi^{\prime}(\alpha)$. Thus $M_{\varphi(\alpha)} \neq M_{\varphi^{\prime}(\alpha)}$, and therefore, by a trivial argument, $\mathscr{G}_{\varphi} \neq \mathscr{G}_{\varphi^{\prime}}$.

The following corollary follows Theorem 3 immediately.

Corollary 1. Let $s$ denote the cardinality of set $\left\{I \mid R_{\mathscr{N}} \subseteq I \subseteq \mathscr{G}, I\right.$ is a norm closed two-sided ideal of $\operatorname{Alg} \mathscr{N}\}$. Then

(1) If $\operatorname{ind}(\alpha)=0$, for every $\alpha \in \Lambda$, then $s=2^{\Lambda}$, where $2^{\Lambda}$ denotes the cardinality of set which consists of all subsets of $\Lambda$.

(2) If ind $(\alpha)=1$, for every $\alpha \in \Lambda$, then $s=3^{\Lambda}$, where $3^{\Lambda}$ denotes the cardinality of the set which consists of all maps from $\Lambda$ to $\{0,1,2\}$. Particularly, if $\bar{\Lambda}=n$ (i.e. the cardinality of $\Lambda$ ) and $\operatorname{ind}(\alpha)=1$ for every $\alpha \in \Lambda$, then $s=3^{n}$.

(3) If $\bar{\Lambda}=n, \bar{\Lambda}_{0}=\{\alpha \mid \operatorname{ind}(\alpha)=0\}, \bar{\Lambda}_{1}=\{\alpha \mid \operatorname{ind}(\alpha)=1\}$, and $\bar{\Lambda}_{0}=m$, $\bar{\Lambda}_{1}=n-m$, then $s=2^{m} \cdot 3^{n-m}$.

\section{ACKNOWLEDGMENT}

The authors are very grateful to the referee for providing a short proof of the Theorem 2 and some other suggestions. 


\section{REFERENCES}

1. Alan Hopenwasser and Divid Larson, The carrier space of a reflexive operator algebra, Pacific J. Math. 81 (1979), 417-434.

2. E. C. Lance, Some properties of nest algebra, Proc. London Math. Soc. 19 (1969), 45-68.

3. D. R. Larson, Nest algebras and similarity transforms, Ann. of Math. (2) 121 (1985), 409-427.

4. J. R. Ringrose, On some algebras of operators, London Math. Soc. 15 (1965), 61-83.

Department of Mathematics, Qufu Normal University, Qufu, Shandong 273165, People's Republic of China 